\title{
Asymmetrical Triangular Current Mode (ATCM) for Bidirectional High Step Ratio Modular Multilevel dc-dc Converter
}

\author{
C. Pineda, Student Member, IEEE, J. Pereda, Member, IEEE, F. Rojas, Member, IEEE, \\ C. Cerda, X. Zhang, Member, IEEE, A. J. Watson Member, IEEE
}

\begin{abstract}
DC networks have proven advantages in high voltage (HVDC) transmission systems, and now they are expanding to medium and low voltage distribution networks. One of the major challenges is to develop reliable dc-dc voltage transformation achieving high efficiency and performance, especially at high voltage and high step ratio. New resonant modular multilevel topologies have arisen as an alternative, mainly because of advantages such as optional use of transformers, natural voltage balance, simple control and soft-switching capability. However, this type of operation generates a high peak current, does not allow control of power flow in all power range and has a limited range of voltage variation. This paper proposes an asymmetrical triangular current mode (ATCM) applied to high step ratio Modular Multilevel dc-dc Converters. The proposed modulation increases the efficiency and achieves bidirectional control of the power, soft-switching and a natural balance of the voltage in the cell capacitors. The experimental results show the bidirectional operation and the capacitor voltage balance of the converter under different operating conditions with higher efficiency $(97.72 \%)$ and lower peak current compared to previous reports of this topology using resonant operation.
\end{abstract}

Index Terms-dc-dc conversion, modular multilevel converter, high step ratio conversion, triangular current mode.

\section{INTRODUCTION}

D IRECT current has important advantages over alternating current in the transmission of electrical energy, such as lower power losses, less and smaller cables and absent of reactive power nor peak voltages. However, these advantages have not been enough for the massive implementation of $\mathrm{dc}$ power systems due to the lack of competitive dc technology, especially high voltage dc-dc converters. The exception is the mature current source converter for high voltage direct current (HVDC) systems, which has demonstrated to be an

Manuscript received July 07, 2019; revised October 16, 2019; accepted November 27, 2019. The original version of this paper was presented at the IEEE Energy Conversion Congress \& Expo 2018, September 17-23, 2018 in Portland, OR, USA. This work was supported by the Chilean Research Council CONICYT under Grant FONDECYT 1171142, FONDECYT Iniciacion 11190806 and SERC Chile (CONICYT/FONDAP/15110019).

C. Pineda and J. Pereda are with the Electrical Engineering Department and the UC Energy Research Center, Pontificia Universidad Católica de Chile, Santiago, Chile, (e-mail: cnpineda@uc.cl; jepereda@ing.puc.cl).

F. Rojas and C. Cerda is with Universidad de Santiago de Chile, Santiago, Chile. (e-mail: felix.rojas@usach.cl; carlos.cerda.j@usach.cl).

$\mathrm{X}$. Zhang is with the School of Electrical Engineering, Xi'an Jiaotong University, Xi'an, 710049, China (e-mail: xiaotian@xjtu.edu.cn).

A. J. Watson is with the Power Electronics Machines and Control Group, University of Nottingham, Nottingham, United Kingdom (e-mail: Alan.Watson@ nottingham.ac.uk). economically and technically attractive option, mostly in specific point-to-point transmission systems such as offshore wind farms and long transmission lines [1]. Nowadays, the voltage source converter (VSC) in HVDC systems has arisen with the use of the modular multilevel converter (MMC) [2], because it introduces the capability to compensate reactive power, ac voltage control, black-start functionality, and multi-terminal connection allowing large meshed and radial HVDC networks [3], [4]. On the other hand, the development of medium and low voltage direct current (MVDC-LVDC) networks is also of great interest due to the increase in dc loads and dc generation sources such as photovoltaic solar panels, electric vehicles and battery energy storage systems. In this scenario, the dc-dc conversion that allows the interconnection of HVDC, MVDC and LVDC systems is a key element for the development of the networks of the future.

A dc-dc converter for $\mathrm{HV}$ and MV range should provide high efficiency, bidirectional power flow, voltage regulation and fault blocking capability [5], [6]. Several dc-dc converter topologies have been proposed, but there are two converter technologies that stand out for their high reliability, modularity and flexibility: the input-series output-parallel (ISOP) converters, based on dc-dc building blocks [7]-[12]; and the modular multilevel front-to-front (MMC-FTF) converters [13]-[19].

The FTF-MMC uses two MMCs connected through a transformer and operates at medium-frequency to reduce the size of the transformer and the passive elements of the converter [14]. This solution provides full bidirectional power flow control and galvanic isolation, but it requires a complex voltage control and hard-switching operation that reduce efficiency, especially in high step ratio voltage transformation.

To solve this problem, resonant MMC topologies have been proposed for high step voltage ratio [20]-[23], adding soft-switching operation to decrease the power losses. These proposals used a resonant tank formed by the capacitors of the cells which is excited by a square wave voltage at the resonant frequency, achieving zero-current-switching (ZCS) in the semiconductor devices. The voltage gain can be controlled changing the number of cells used in the stack modulation [21], [22], but it is more suitable for reconfiguration or for a degraded operation rather than for accurate voltage control due to its step behaviour. Thus, control through the variation of the operating frequency has been the most employed technique [20], [24], allowing precise control of transferred power. However, operation at a frequency different than the 
resonant frequency is more complex to model and the ZCS is lost, limiting the power range in which the converter is highly efficient. Also, the resonant operation is highly sensitive to the parameters of the resonant tank, requires a design that carefully considers parasitic elements, and generates high peak current in the ac link. This high peak current cause high conduction losses and low efficiency despite its soft-switching capability.

Alternatively, triangular current mode (TCM) scheme applied to dual active bridge (DAB) dc-dc converters [25], [26] achieves bidirectional control of the power maintaining ZCS operation without resonance. Therefore, the design process and control scheme are simpler than resonant operation, extending high-efficiency operation to full power range. Also, the bidirectional operation using TCM has a symmetrical gain in both directions of the power flow. In the resonant case, it needs a symmetric resonant tank as PI type or T type LCL/CLC resonant tank to get a symmetric gain [27], [28]. However, TCM has not been applied in MMC topologies because it is not compatible in a single-pole configuration.

This paper presents a new asymmetrical triangular current mode (ATCM) for high step ratio dc-dc MMC, which achieves bidirectional power flow through a simple control law, maintains ZCS operation, reduces the peak current compared to resonant mode, and presents natural balance of the voltage in the cell capacitors without the need of complex control strategies [29]. The high step ratio dc-dc MMC topology is presented in Section II, the proposed modulation of the converter is described in Section III and the dynamic balance of power is analysed in Section IV. Finally, the simulation and experimental results are shown in Section V.

\section{High Step Ratio Modular Multilevel DC-DC CONVERTER}

The topology of the high step ratio dc-dc MMC is shown in Fig. 1. The high-voltage port contains a stack of $\mathrm{N}$ halfbridge cells connected through an inductor to an active fullbridge converter, enabling the bidirectional control power flow between the high-voltage side $\left(V_{H V}\right)$ and the low-voltage side $\left(V_{L V}\right)$. A transformer could be optionally used between the inductor and the full-bridge to provide galvanic isolation and a higher step ratio. Additionally, filters are connected in high-voltage and low-voltage side to decrease voltage and peak current, but they are not considered in the analysis for simplicity.

The dynamic equations that describe the general operation of the converter consider the voltages at both side of the inductor $v_{L 1}$ and $v_{L 2}$ as is shown in (1)-(2). The voltage $v_{L 1}$ is the difference between the voltage $V_{H V}$ and the voltage in the half-bridge stack $v_{\text {stack }}$. The voltage $v_{\text {stack }}$ is the sum of all voltages in the half-bridge cells $v_{i}$, therefore it can be defined in terms of the commutation state $S_{i-H B} \in\{1,0\}$ and the capacitor voltage $v_{c i}$ of each cell $\left(v_{\text {stack }}=\sum_{i=1}^{N} v_{c i} \cdot S_{i-H B}\right)$. On the other hand, the voltage $v_{L 2}$ is determined by the full-bridge commutation state $S_{F B} \in\{1,0,-1\}$ and the low voltage $V_{L V}\left(v_{L 2}=V_{L V} \cdot S_{F B}\right)$. The inductor current $i_{L}$, the commutation state $S_{i-H B}$ and the capacitance $C_{i}$ of each

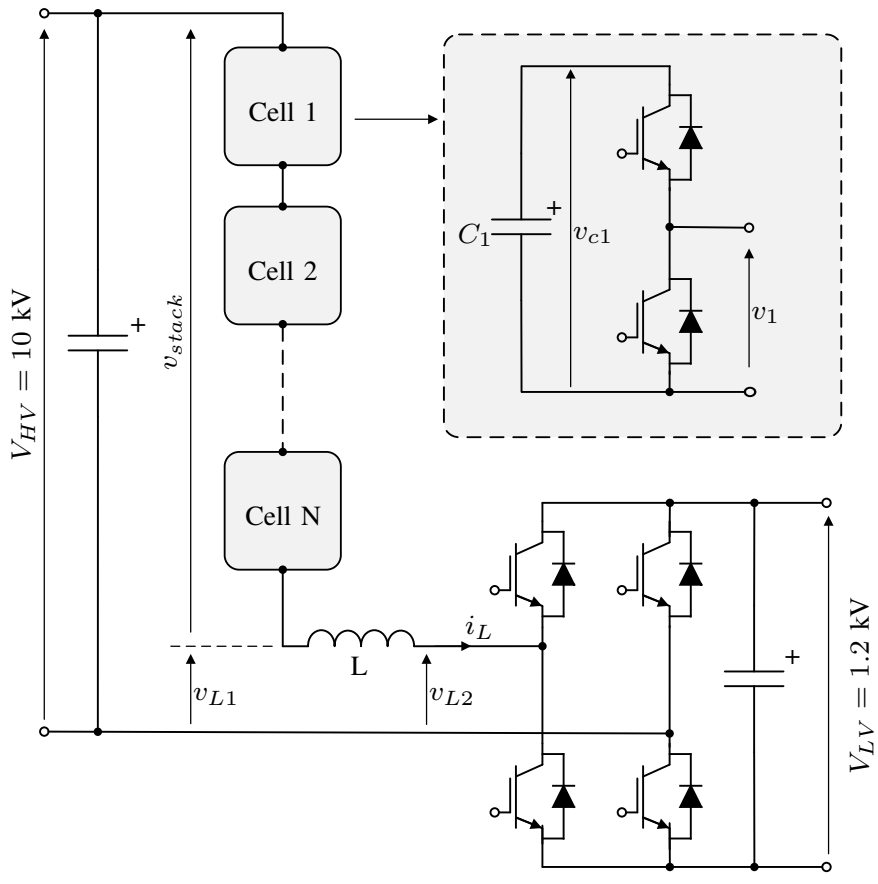

Fig. 1. Topology of the high step ratio dc-dc MMC using half-bridges in cells.

cell capacitor defines the capacitor voltage waveform $v_{c i}$ in each half-bridge cell (2), determining their ripple, mean value and dynamic behaviour. The resistance $r_{e q}$ represents the total conduction loss resistance in the converter generated by the inductor, switches, capacitors and wire.

$$
\begin{gathered}
L \frac{d i_{L}}{d t}=\underbrace{V_{H V}-\overbrace{\sum_{i=1}^{N} v_{c i} \cdot S_{i-H B}}^{v_{\text {stack }}}}_{v_{L 1}}-i_{L} \cdot r_{e q}-\underbrace{V_{L V} \cdot S_{F B}}_{v_{L 2}} \\
C_{i} \frac{d v_{c i}}{d t}=i_{L} \cdot S_{i-H B}
\end{gathered}
$$

The inductor current $i_{L}$ is controlled through the modulation of the voltages $v_{L 1}$ and $v_{L 2}$. And the direction and magnitude of power are controlled directly with the inductor current.

\section{Proposed Modulation Technique}

The triangular control mode (TCM) requires a coordinated three-level voltage in both terminals of the inductor, $v_{L 1}$ and $v_{L 2}$. The full-bridge generates three-levels in $v_{L 2}$ by using unipolar modulation, but the stack requires a special modulation to get three-levels in $v_{L 1}$. The following subsection III-A presents the proposed stack modulation. Then, subsection III-B presents the triangular current mode proposal.

\section{A. Half-Bridges Stack Modulation}

The proposed modulation in the stack is aimed to generate a three-level voltage $v_{L 1}$ controlled by transitions times $t_{1}, t_{3}$, 


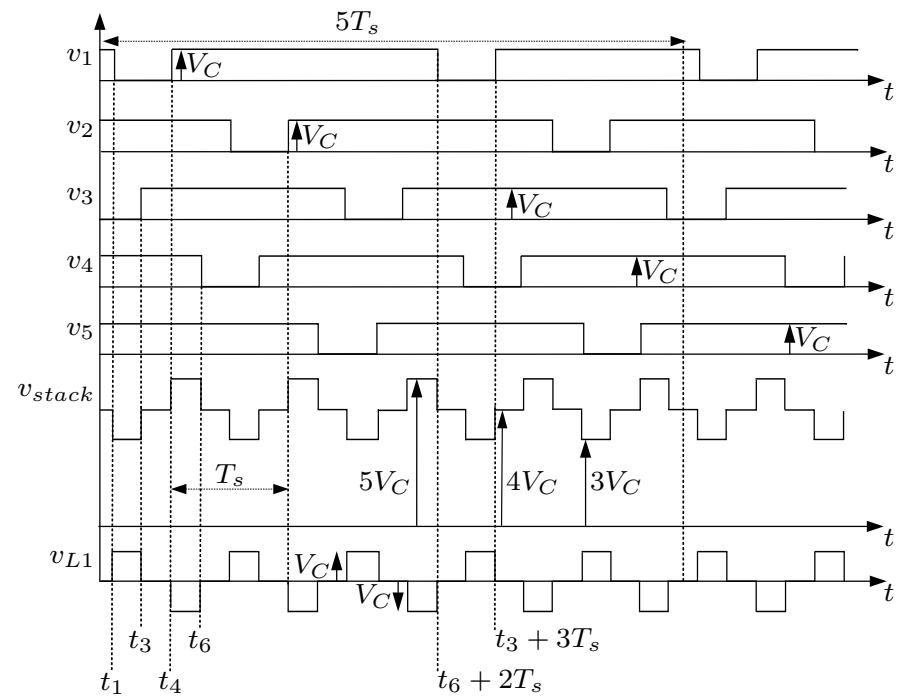

Fig. 2. Operation waves in high voltage side for $\mathrm{N}=5$ cells and $\mathrm{j}=2$.

$t_{4}$ and $t_{6}$ as is shown in Fig. 2, extending the modulation presented in [23] to an asymmetric operation. In order to achieve this, the cells are modulated with the same waveform using a phase shift of $T_{S}$ to reduce the resultant switchingfrequency and to achieve a balanced use of the cells. The commutation state of the first half-bridge cell $S_{1}^{j}$ is considered as a periodical signal of period $N \cdot T_{s}$ defined by transition times and a free parameter $j$ as is shown in (3). $j$ is a free parameter that defines a set of different cell waveforms in the half-bridge cell that cause the same voltage in the stack if $j \in \mathbb{N} \mid(0 \leq j<N-1)$.

$$
S_{1-H B}^{j}(t)= \begin{cases}0 & \text { if } t_{1}<t<t_{4} \\ & t_{6}+j \cdot T_{s}<t<t_{3}+(j+1) T_{s} \\ 1 & \text { else }\end{cases}
$$

Therefore, a time-controlled voltage $v_{\text {stack }}$ of three-levels centred on $(N-1) V_{C}$, amplitude $V_{C}$ and period $T_{s}$ is generated in the stack if the voltage in the cells are phase shifted by $T_{s}$ and all the capacitor voltages in the cells are balanced and equal to $V_{C}$, as is determined in (4).

$$
v_{\text {stack }}= \begin{cases} & 0<t<t_{1} \\ (N-1) V_{C} & t_{3}<t<t_{4} \\ & t_{6}<t<T_{s} \\ & \\ (N-2) V_{C} & t_{1}<t<t_{3} \\ N \cdot V_{C} & t_{4}<t<t_{6}\end{cases}
$$

Finally, a voltage $v_{L 1}$ of three-levels centred on zero, amplitude $V_{C}$, period $T_{s}$ and controlled by transition times $t_{1}, t_{3}, t_{4}$ and $t_{6}$ is obtained if the relation (5) is achieved, as is shown in (6).

$$
V_{C}=\frac{V_{H V}}{(N-1)}
$$

$$
v_{L 1}= \begin{cases} & 0<t<t_{1} \\ & t_{3}<t<t_{4} \\ & t_{6}<t<T_{s} \\ V_{C} & t_{1}<t<t_{3} \\ -V_{C} & t_{4}<t<t_{6}\end{cases}
$$

The proposed modulation waveforms for each cell is illustrated in Fig. (2), taking $N=5$ and $j=2$ as an example. The successful operation relies on all cells being healthy. However, in case of failure, it is possible to bypass the faulty cell and adjust the $V_{C}$ to the new number of cells.

\section{B. Asymmetrical Triangular Current Mode (ATCM)}

Considering the stack modulation previously presented and neglecting the equivalent resistance $r_{e q}$, the dynamic equation (1) can be simplified to (7). This model is equivalent to a DAB linked by an inductor, allowing the use of the triangular current mode (TCM) [25]. TCM controls the power flow through the transition times of the voltages on both sides of the inductor. Therefore, the slope of the inductor current $i_{L}$ is defined by the inductance $L$ and its voltage, which is imposed by the difference between the voltages controlled by the stack $v_{L 1}$ and the full-bridge $v_{L 2}(7)$. The main advantages of the TCM are that it operates with soft-switching in most semiconductor transitions, and it can limit the peak current.

$$
L \frac{d i_{L}}{d t}=v_{L 1}-v_{L 2}
$$

Conventional TCM generates a symmetric current in the inductor, which is desired for DAB converters, but it is a problem for the proposed topology. A symmetric current would generate zero power flow from the high-voltage side because the stack is connected in series with the high-voltage dc-link. To solve this problem, an asymmetric triangular current mode (ATCM) scheme is proposed to achieve bidirectional control of the power and to maintain the ZCS. The proposed ATCM scheme is illustrated in Fig. 3.

During the intervals $\left(0, t_{1}\right),\left(t_{3}, t_{4}\right)$ and $\left(t_{6}, T_{s}\right)$, the voltages $v_{L 1}$ and $v_{L 2}$ are zero to keep the current $i_{L}$ constant and equal to zero. In $t_{1}$, a cell in the stack is bypassed generating $V_{C}$ voltage in $v_{L 1}$ while the full-bridge is kept in zero state (Fig. 3 (a)). The voltage $V_{C}$ is therefore applied to the inductor, causing the current $i_{L}$ to increase linearly. In $t_{2}$, the full-bridge imposes the low-voltage $\left(V_{L V}\right)$ in $v_{L 2}$, so the inductor voltage is defined by the difference of the voltages applied by both converters $\left(V_{C}-V_{L V}\right)$. Therefore, the low-voltage $V_{L V}$ must be greater than $V_{C}$ in order to obtain a negative slope of the inductor current and get zero current switching at $t_{3}$. When the current reaches zero in $t_{3}$, an additional cell module is connected generating zero voltage in $v_{L 1}$ and the full-bridge is switched applying zero voltage in $v_{L 2}$. Thus, the equations for current $i_{L}$ in positive power flow are show in (8). 


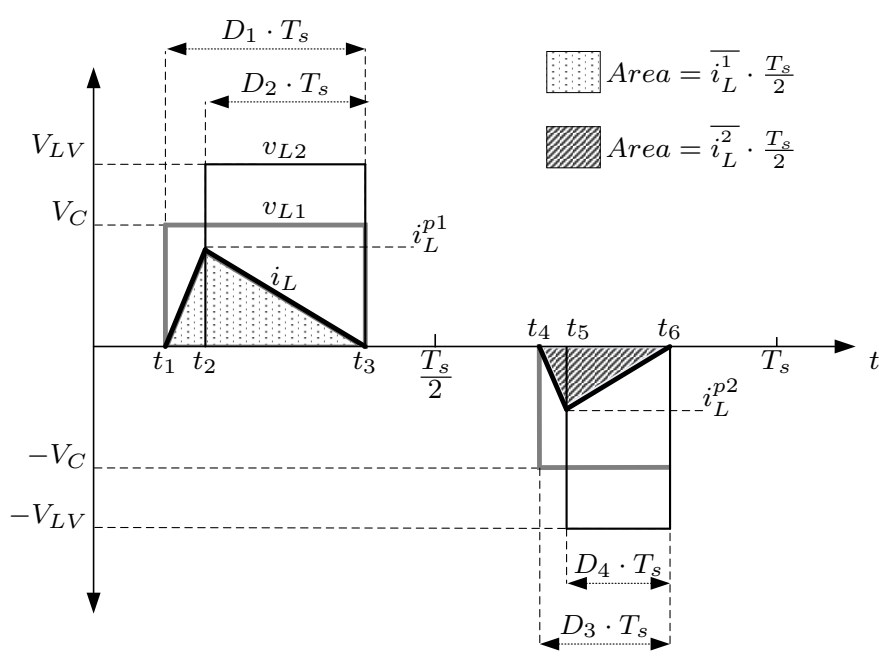

(a)

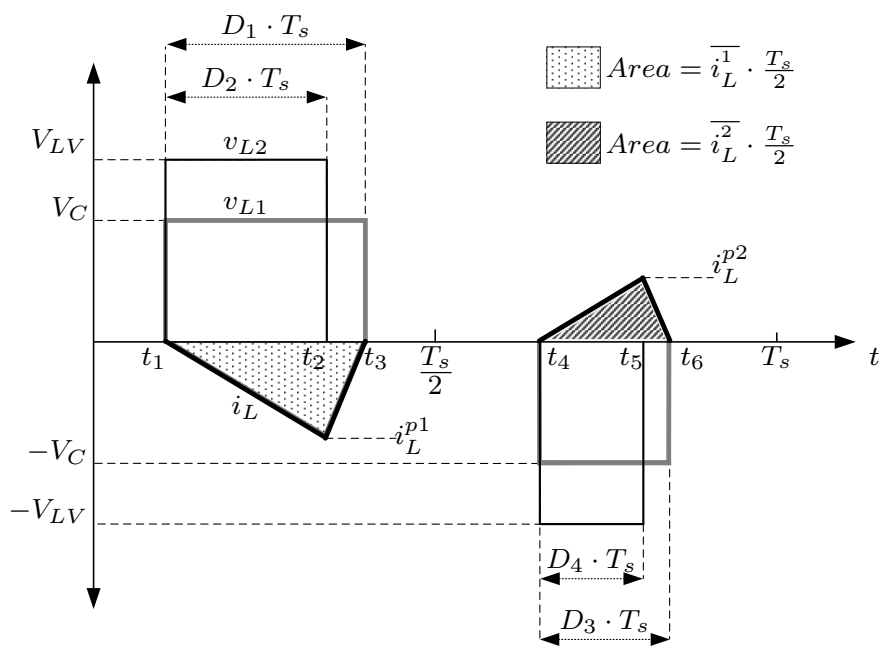

(b)

Fig. 3. Current and voltage waveforms for converter under ATCM and ZCS: (a) power flow from high-voltage side to low voltage side. (b) power flow from low-voltage side to high-voltage side.

$$
i_{L}(t)= \begin{cases}0 & \text { if } t \in\left(0, t_{1}\right),\left(t_{3}, t_{4}\right) \\ \frac{V_{C}}{L}\left(t-t_{1}\right) & \text { if } t \in\left(t_{1}, t_{2}\right) \\ i\left(t_{2}\right)-\frac{V_{L V}-V_{C}}{L}\left(t-t_{2}\right) & \text { if } t \in\left(t_{2}, t_{3}\right) \\ -\frac{V_{C}}{L}\left(t-t_{4}\right) & \text { if } t \in\left(t_{4}, t_{5}\right) \\ i\left(t_{5}\right)+\frac{V_{L V}-V_{C}}{L}\left(t-t_{5}\right) & \text { if } t \in\left(t_{5}, t_{6}\right)\end{cases}
$$

ZCS restrictions are determined in (9) considering duty cycles $D_{i}\left(0<D_{i} \leq 0.5\right)$ as in Fig. 3 (a), the conditions for continuity of the current $i_{L}$ for $t_{2}$ and $t_{5}$ times, and imposing that the current reach zero in $t_{3}$ and $t_{6}$ times in (8).

$$
D_{i+1}=\left(\frac{V_{C}}{V_{L V}}\right) D_{i} \quad i=1,3
$$

Therefore, peak currents in the inductor $i_{L}^{p 1}$ and $i_{L}^{p 2}$ can be found in (10), using ZCS restrictions (9) and noticing that these occur in the $t_{2}$ and $t_{5}$ times.

$$
\begin{aligned}
& i_{L}^{p 1}=i_{L}\left(t_{2}\right)=\frac{V_{C}}{f_{s} L}\left(1-\frac{V_{C}}{V_{L V}}\right) D_{1} \\
& i_{L}^{p 2}=i_{L}\left(t_{5}\right)=-\frac{V_{C}}{f_{s} L}\left(1-\frac{V_{C}}{V_{L V}}\right) D_{3}
\end{aligned}
$$

\section{DynAmic BALANCE OF POWER}

The previous analysis requires that the mean voltage in the capacitors of the cells maintains a constant value during their operation. This section presents the voltage balance condition in the converter and a simple model to control the power transfer between ports maintaining this balance.

\section{A. Voltage in the cell capacitors}

Considering the ATCM and the proposed modulation, the voltage in each capacitor cell can be found integrating (2). The capacitor voltage at the end of the modulation time window $N \cdot T_{s}$ can be expressed using the peak currents as is shown in (11).

$$
\begin{aligned}
v_{c i}\left(t_{0}+N \cdot T_{s}\right)= & v_{c i}\left(t_{0}\right)+ \\
& \frac{i_{L}^{p 1} \cdot(N-2) \cdot D_{1}+i_{L}^{p 2} \cdot N \cdot D_{3}}{2 f_{s} C_{i}}
\end{aligned}
$$

In order to achieve a steady state balance in the voltage capacitors, this voltage must remain in the same value at the end of the modulation time window. Therefore, the steady state voltage balance condition can be found in (12).

$$
D_{3}=D_{1} \sqrt{\frac{N-2}{N}}
$$

The ratio $\frac{D_{3}}{D_{1}}$ represents the level of asymmetry of the current in the inductor. Also, the asymmetry needed to keep the steady-state capacitors voltage balance decreases as the number of cells used increases.

The peak to peak voltage in the capacitors $v_{c i}^{p p}$ in steady state depend of their capacitance, peak currents and number of cells. Therefore, a capacitance value to keep the voltage ripple within a certain boundary can be obtained from (13) using the worse condition $\left(D_{1}=0.5\right)$.

$$
v_{c i}^{p p}=\frac{i_{L}^{p 1} \cdot D_{1}(N-2)}{N f_{s} C_{i}}
$$

\section{B. Power transfer}

In order to calculate the power transferred between the highvoltage port, the low-voltage port and the interchange of power among the cells, the average inductor currents in the first $\left(\overline{i_{L}^{1}}\right)$ and second $\left(\overline{i_{L}^{2}}\right)$ half cycles of the switching cycle are defined in (14) and illustrated in Fig. 3 (a). These average currents can be defined as a function of the peak currents in the inductor $i_{L}^{p 1}$ and $i_{L}^{p 2}$ using the proposed ATCM, however this analysis 
will consider a generalized inductor current waveform at this point.

$$
\begin{aligned}
& \overline{i_{L}^{1}}=\frac{2}{T_{s}} \int_{0}^{\frac{T_{s}}{2}} i_{L} \cdot d t \\
& \overline{i_{L}^{2}}=\frac{2}{T_{s}} \int_{\frac{T_{s}}{2}}^{T_{s}} i_{L} \cdot d t
\end{aligned}
$$

The average power delivered by the high voltage port in one switching cycle $P_{H V}$ is defined by the sum of the average currents $\overline{i_{L}^{1}}$ and $\overline{i_{L}^{2}}$ due to the inductor current waveform is the same as in the high voltage port if filters are not considered. On the other hand, the average power in the inductor $P_{L V}$ is defined by its difference as is described in (15). The same capacitor voltage balance condition in the cell stack (12) is found through these equations considering ATCM and neglecting the power losses $\left(P_{H V}=P_{L V}\right)$. This extends the voltage capacitor balance analysis to other modulations in the stack such as sorting modulation.

$$
\left[\begin{array}{c}
P_{H V} \\
P_{L V}
\end{array}\right]=\left[\begin{array}{c}
V_{H V} \\
V_{C}
\end{array}\right]\left[\begin{array}{cc}
\frac{1}{2} & \frac{1}{2} \\
\frac{1}{2} & -\frac{1}{2}
\end{array}\right]\left[\begin{array}{l}
\overline{i_{L}^{1}} \\
\overline{i_{L}^{2}}
\end{array}\right]
$$

Alternatively, (15) can be expressed as a function of the average power for the stack $P_{\text {stack }}$ in (16), due it is the difference of these two average power $\left(P_{\text {stack }}(k)=P_{H V}-P_{L V}\right)$ if the cell power losses are neglected.

$$
\left[\begin{array}{c}
P_{H V} \\
P_{\text {stack }}
\end{array}\right]=\left[\begin{array}{cc}
\frac{V_{H V}}{2} & \frac{V_{H V}}{2} \\
\frac{V_{H V}-V_{C}}{2} & \frac{V_{H V}+V_{C}}{2}
\end{array}\right]\left[\begin{array}{l}
\overline{i_{L}^{1}} \\
\overline{i_{L}^{2}}
\end{array}\right]
$$

Thus, using (15) or (16) is possible to control the power transfer between high voltage and low voltage ports through the average currents $\overline{i_{L}^{1}}$ and $\overline{i_{L}^{2}}$. The control law for the power flow $P_{H V}$ is determined in (17), considering the ATCM lossless model in steady state. $D_{1}$ is able to control the power transfer and the maximum power $P_{H V}^{\max }$ is obtained evaluating $P_{H V}$ at $D_{1}=0.5$. A similar analysis can be performed for negative power flow inverting the phase shift of voltages $v_{L 1}$ and $v_{L 2}$ as is illustrated in Fig 3 (b). Therefore, these relationships allow full bidirectional control of the power flow whilst maintaining the ZCS and voltage balance in the converter.

$$
\begin{aligned}
P_{H V} & = \pm 4 D_{1}^{2} P_{H V}^{\max } \\
P_{H V}^{\max } & =\frac{(N-1) V_{C}^{2}\left(V_{L V}-V_{C}\right)}{4 N f_{s} L \cdot V_{L V}}
\end{aligned}
$$

The resistive elements in the inductor $\left(r_{l}\right)$, switches $\left(r_{s w}\right)$ and capacitors $\left(r_{c}\right)$ reduce the maximum power transfer due conduction losses and voltages drop at both sides of inductor. This voltage drop can be incorporated in the analysis of power transfer, considering the equivalent resistance $r_{e q}$ as the sum of all resistance elements in the converter, as is shown in (18). Two switches in the full-bridge and $\mathrm{N}$ cell switches in the stack are conducting in any commutation state. Also, the number of
TABLE I

RESONANT OPERATION AND ATCM COMPARISON

\begin{tabular}{lcc}
\hline \hline Parameter & Resonant Operation & ATCM \\
\hline Maximum peak current & $\frac{\pi}{2}\left(2 N-1+\frac{2}{\pi}\right) \frac{P_{H V}^{\max }}{V_{H V}}$ & $2 N \frac{P_{H V}^{\max }}{V_{H V}}$ \\
Stack voltage ratio & $\frac{2}{2 N-1}$ & $\frac{2}{N-1}$ \\
\hline \hline
\end{tabular}

capacitors connected can be approximated by a mean value of $(N-1)$ since the number of capacitors inserted using the proposed modulation is alternating between $(N-2),(N-1)$ and $N$.

$$
r_{e q}=r_{l}+(N+2) \cdot r_{s w}+(N-1) \cdot r_{c}
$$

Therefore, the power transfer considering voltage drop due resistive elements $P_{H V}^{r}$ can be found solving (19).

$$
P_{H V}^{r}=\frac{D_{1}^{2}(N-1) V_{C}^{2}\left(V_{L V}-V_{C}-\frac{P_{H V}^{r} \cdot r_{e q}}{V_{H V}}\right)}{\left(N f_{s} L \cdot V_{L V}-\frac{P_{H V}^{r} \cdot r_{e q}}{V_{H V}}\right)}
$$

The main benefit of ATCM over the alternative resonant operation is a simpler model that works across the entire power range without losing ZCS. The resonant operation requires modifying the operating frequency to control the power transferred between the ports, making the model more complex and losing the ZCS at operating frequencies other than the resonant frequency. Alternatively, the ATCM model is valid in the entire power range with ZCS, making the converter highly efficient in any operation point. Also, the design process is easier and less restrictive due to the fixed frequency operation and the absence of the resonant tank highly dependent of parasitic elements or variations of cell capacitance.

The maximum peak current and stack voltage ratio $\left(\frac{v_{L 1}^{p p}}{V_{H V}}\right)$ are compared in Table I, showing a significant reduction in the maximum peak current for equivalent high voltage port and cells rate. However, ATCM generates less stack voltage ratio than the resonant operation because ATCM operates with three-levels voltage in the ac link instead of a two-level voltage. Therefore, the resonant operation achieves a higher voltage ratio at the same number of cells, meanwhile ATCM operates with better use of the dc current component.

\section{RESULTS}

This section presents two sets of results, the simulation in a 1MW full-scale converter, and the experimental results in a scale-down prototype.

\section{A. Real-scale Simulation}

The parameters of the full-scale proposed converter are listed in Table II. Different capacitance values are considered for each capacitor in the cells to evaluate the natural balance under more realistic conditions. 

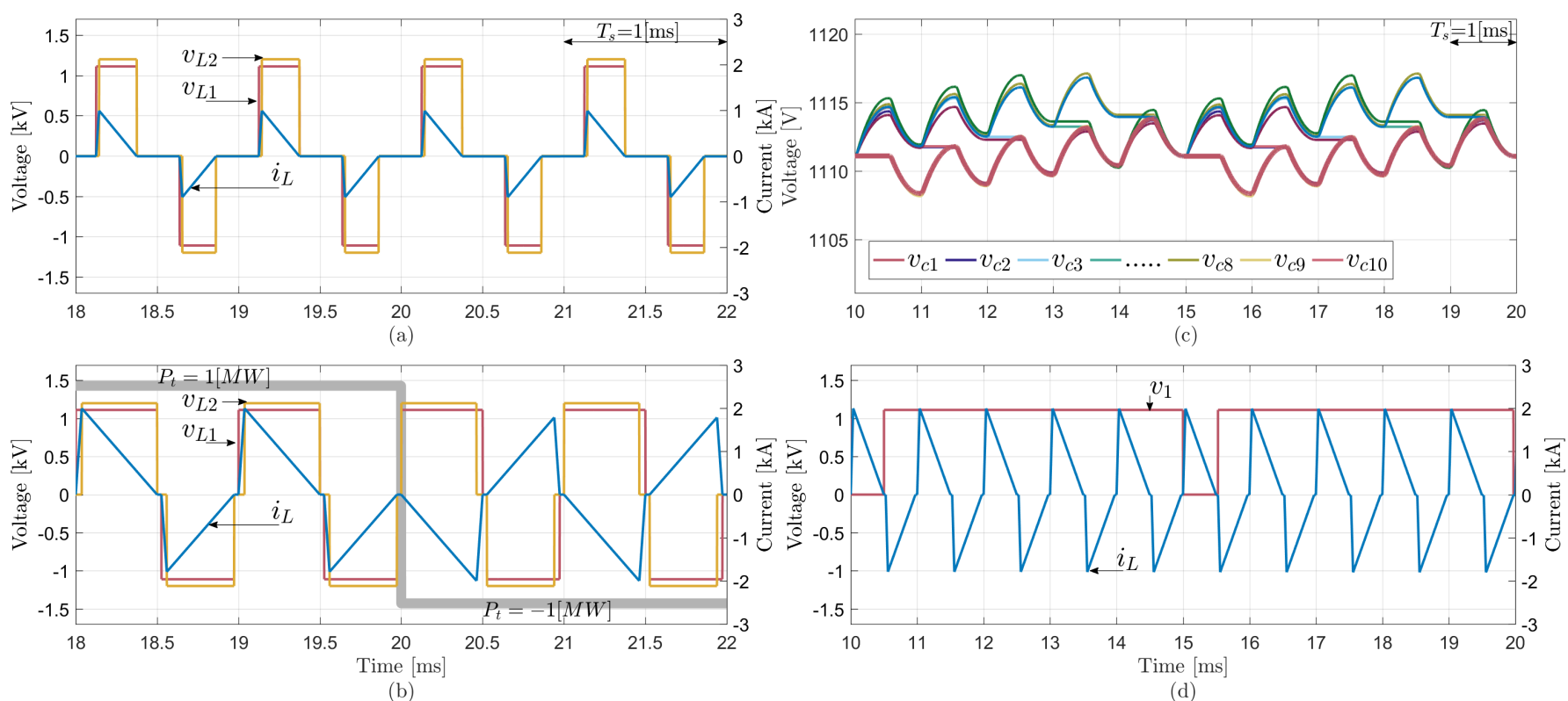

Fig. 4. Simulation waveforms. (a) Voltage and current at $25 \%$ of the maximum power. (b) Voltage and current for maximum power reversal. (c) Waveforms of capacitor cells voltages. (d) Voltage and current of one cell at maximum power.

TABLE II

PARAMETERS OF SIMULATION

\begin{tabular}{lcl}
\hline \hline Description & Parameter & Value \\
\hline Maximum Power & $P_{\text {tmax }}$ & $1 \mathrm{MW}$ \\
HV side Voltage & $V_{H V}$ & $10 \mathrm{kV}$ \\
LV side Voltage & $V_{L V}$ & $1.2 \mathrm{kV}$ \\
Number of cells & $N$ & 10 \\
Inductor inductance & $L$ & $20.6 \mu \mathrm{H}$ \\
Capacitance of cell capacitors & $C_{i}$ & $144 \mathrm{mF} \pm 20 \%$ \\
Switching frequency of the full-bridge & $f_{s}$ & $1 \mathrm{kHz}$ \\
IGBTs ABB 5SNA3600E17000 & $\mathrm{V} / \mathrm{I}$ & $1.7 \mathrm{kV} / 3.6 \mathrm{kA}$ \\
\hline \hline
\end{tabular}

Fig. 4 (a) shows the operation of the converter at $25 \%$ of the maximum power with the low voltage side as load, illustrating the voltages and current of the inductor in four switching periods. Fig. 4 (b) illustrates the step response of the converter for maximum power reversal in the same time window.

The voltages of each cell capacitor in the modulation time window are shown in Fig. 4 (c), verifying the correct power balance of the converter and cells even with different capacitance values for the capacitors of each cell. The ripple voltage in the capacitors is limited to $0.5 \%$ as it was designed.

Voltage and cell current for one cell are shown in Fig. 4 (d), verifying the ZCS in this side of the converter. The voltage $v_{c 1}$ and $v_{1}$ represents the voltage of the capacitor and the output voltage of the cell one respectively. Thus, the charge and discharge process of the capacitor $v_{c 1}$ can be observed in Fig. 4 (c) and (d). Also, the ratio $\frac{N-2}{N}$ used in balance condition (12) can be deducted from Fig. 4 (d) as the number of cycles of positive current over negative current in the cell while it is connected $\left(v_{1}=V_{C}\right)$.

The overall simulated efficiency is over $98.25 \%$ in all power range, as is shown in Fig. 5. The following power losses were taken into account: (i) IGBT conduction losses; (ii) IGBT turnon and turn-off switching losses; (iii) diode reverse recovery losses; and (iv) diode conduction losses. The power losses in the cells stack at full power represents $76.5 \%$ of total losses and are composed only by conduction losses due to the ZCS operation (Fig. 6). The power losses in the low voltage fullbridge at full power represent the other $23.5 \%$ of total losses and are distributed more evenly between conduction $(59.6 \%)$ and switching losses (40.4\%). The IGBTs switching losses in the full-bridge are generated by the only two hard-switching transitions at times $t_{2}$ and $t_{5}$, meanwhile the IGBT conduction losses are negligible because they are only conducting for the short periods $\left(t_{1}, t_{2}\right)$ and $\left(t_{4}, t_{5}\right)$ when the power flow is from high voltage to low voltage port (Fig. 3 (a)). Thus, the full-bridge diodes are conducting most of the time, and their switching losses are almost zero because the transitions are made with ZCS at $t_{3}$ and $t_{6}$ times. The peak current is 2.27 times the average current at full power, and it increases as the power transfer decreases (Fig. 5). Both efficiency and peak current achieve better results with ATCM than those previously presented using a resonant operation for a similarly designed converter [20].

\section{B. Experimental Scale-down Prototype}

The scale-down prototype (Fig. 7) was designed for $1.3 \mathrm{~kW}$ of maximum power transfer between a high voltage side of $950 \mathrm{~V}$ and a low voltage side of $260 \mathrm{~V}$. The high voltage port was generated by a bidirectional dc power supply and the low voltage port by a unidirectional dc power supply, therefore the steady-state experiments consider a power flow from low to high voltage side due the unidirectional power supply limitation. Pre-designed cells were used in the stack, so the device technology (MOSFET) and the sizing are not 


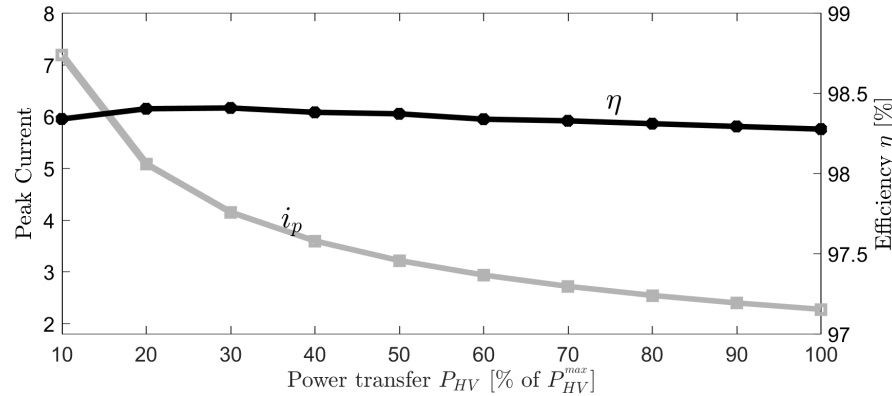

Fig. 5. Simulated power efficiency and peak current for different references of transfer power.

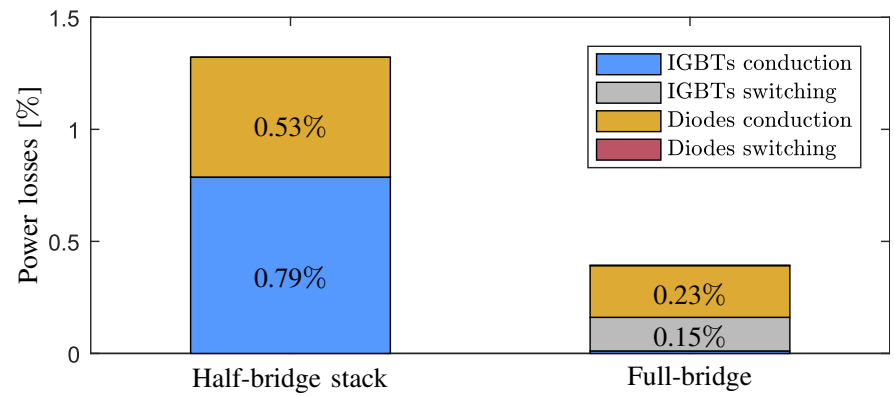

Fig. 6. Power losses distribution at full power transfer.

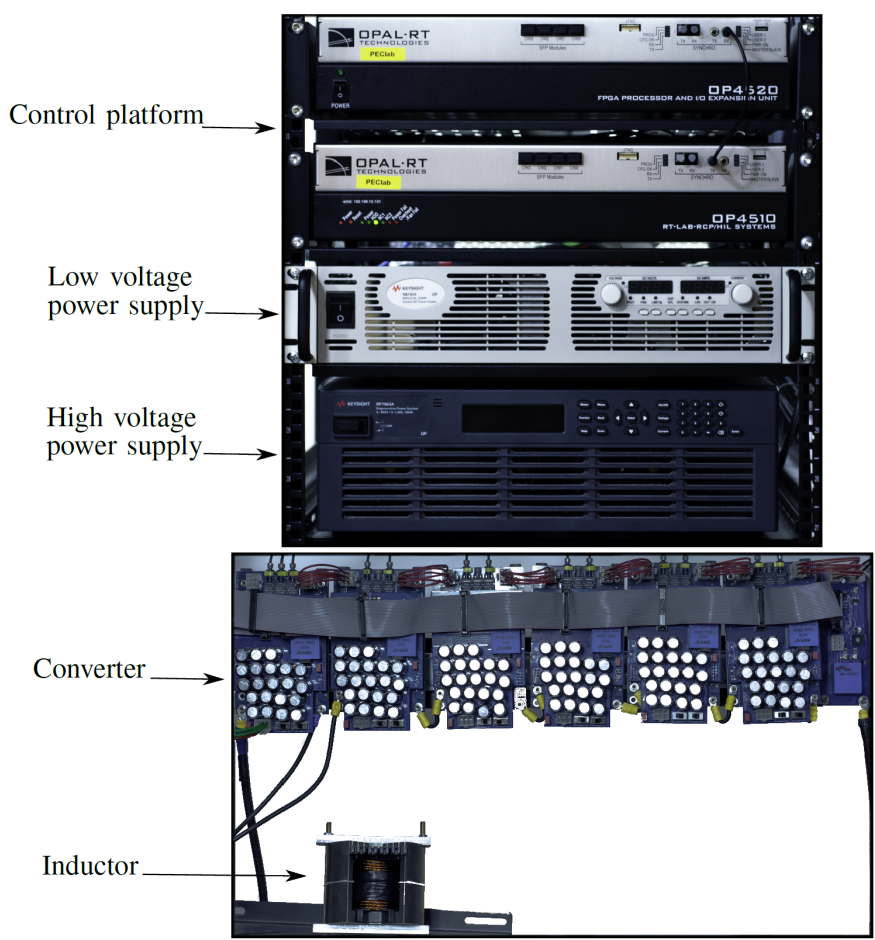

Fig. 7. Experimental scaled-down prototype.

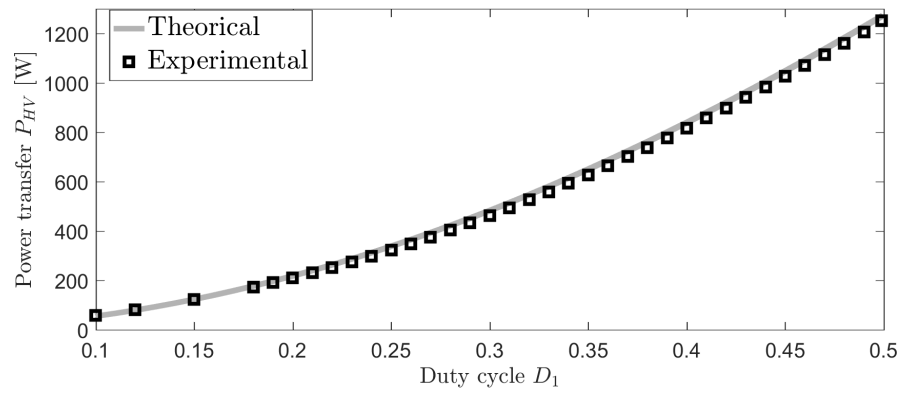

Fig. 8. Experimental power transfer vs duty cycle $D_{1}$.

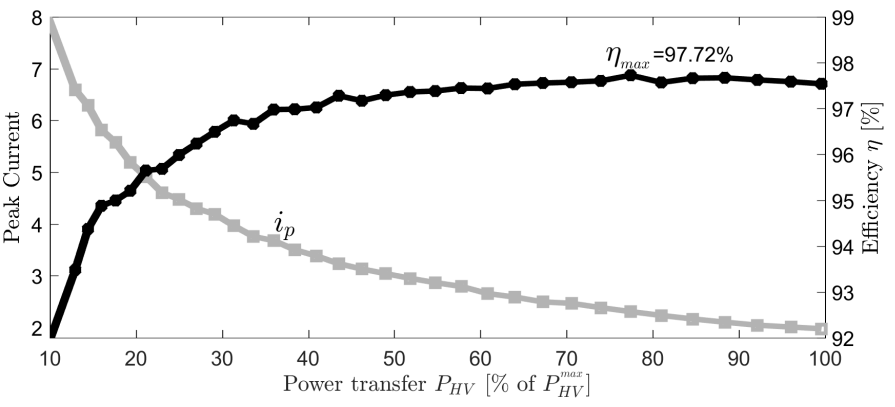

Fig. 9. Experimental power efficiency and peak current for different references of transfer power.

optimal. IGBTs are a better option at this operating frequency because their lower conduction losses take advantage of the ZCS operation. However, the prototype works correctly to validate the theoretical analysis. The inductor was built using a ferrite core, and it was designed to avoid saturation in the core for a maximum peak current of $13 \mathrm{~A}$. The detailed parameters of the prototype are listed in Table III. An OPALRT OP4510/4520 was used as the control platform and to generate the switching signals.

To validate the model, the converter was tested in the full range of power transfer. The experimental results validate the control of the power transfer through the duty cycle $D_{1}$ fitting well to the theoretical model considering the conduction losses from (19) as is shown in Fig. 8. The steady-state waveforms of voltages and current in the inductor at $25 \%$ of power transfer from low to high voltage port show a behaviour concordant with the previous theoretical analysis and simulations, as is illustrated in Fig. 10 (a). The step response of the converter,

TABLE III

PARAMETERS OF EXPERIMENTAL PROTOTYPE

\begin{tabular}{lcl}
\hline \hline Description & Parameter & Value \\
\hline Maximum Power & $P_{t \max }$ & $1.3 \mathrm{~kW}$ \\
HV side Voltage & $V_{H V}$ & $950 \mathrm{~V}$ \\
LV side Voltage & $V_{L V}$ & $260 \mathrm{~V}$ \\
Number of cells & $N$ & 5 \\
Inductor inductance & $L$ & $480 \mu \mathrm{H}$ \\
Capacitance of cell capacitors & $C_{i}$ & $987 \mu \mathrm{F} \pm 20 \%$ \\
Switching frequency of the full-bridge & $f_{s}$ & $1 \mathrm{kHz}$ \\
MOSFETs Infineon IPW65R041CFD & $\mathrm{V} / \mathrm{I}$ & $650 \mathrm{~V} / 80 \mathrm{~A}$ \\
\hline \hline
\end{tabular}



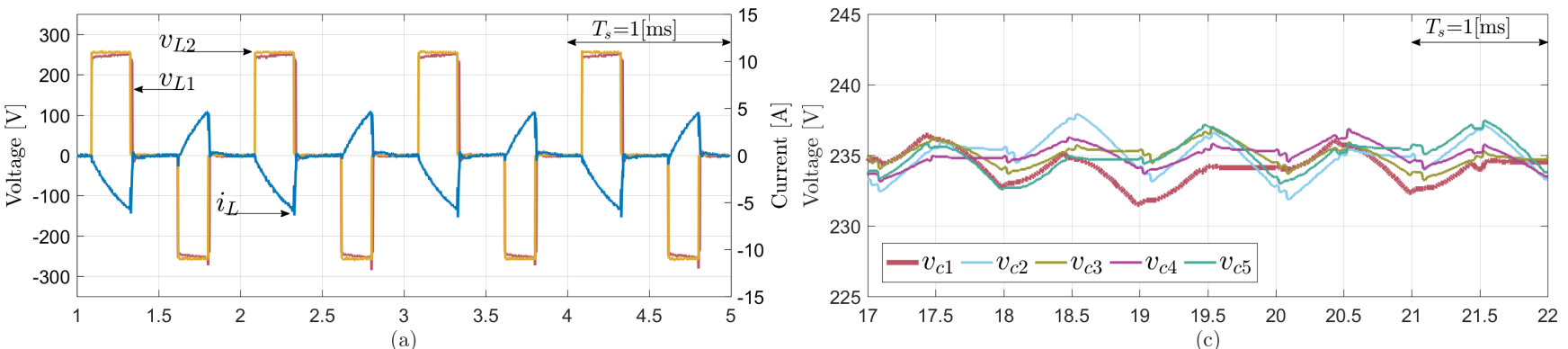

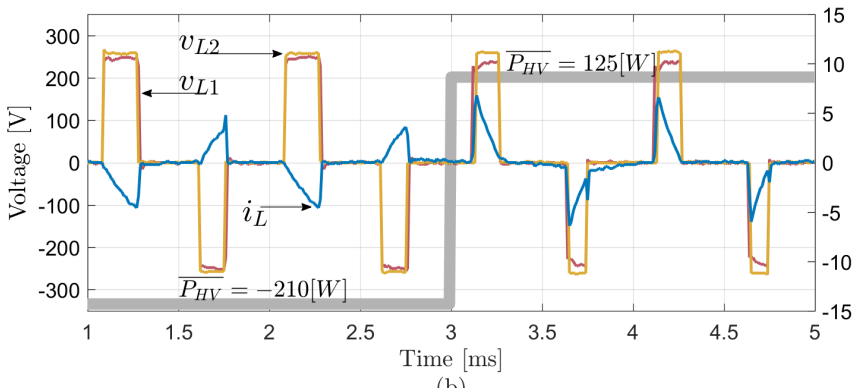

(b)

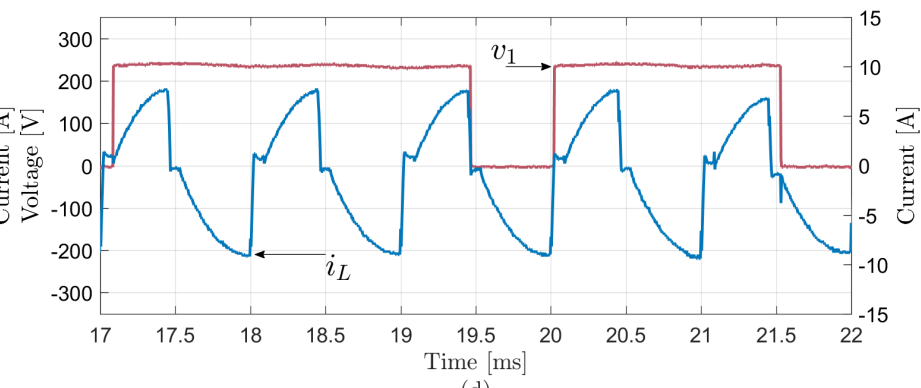

(d)

Fig. 10. Experimental waveforms. (a) Voltage and current at $25 \%$ of the maximum power from low voltage to high Voltage side. (b) Voltage and current for power reversal. (c) Waveforms of capacitor cells voltages at maximum power. (d) Voltage and current of one cell at maximum power.
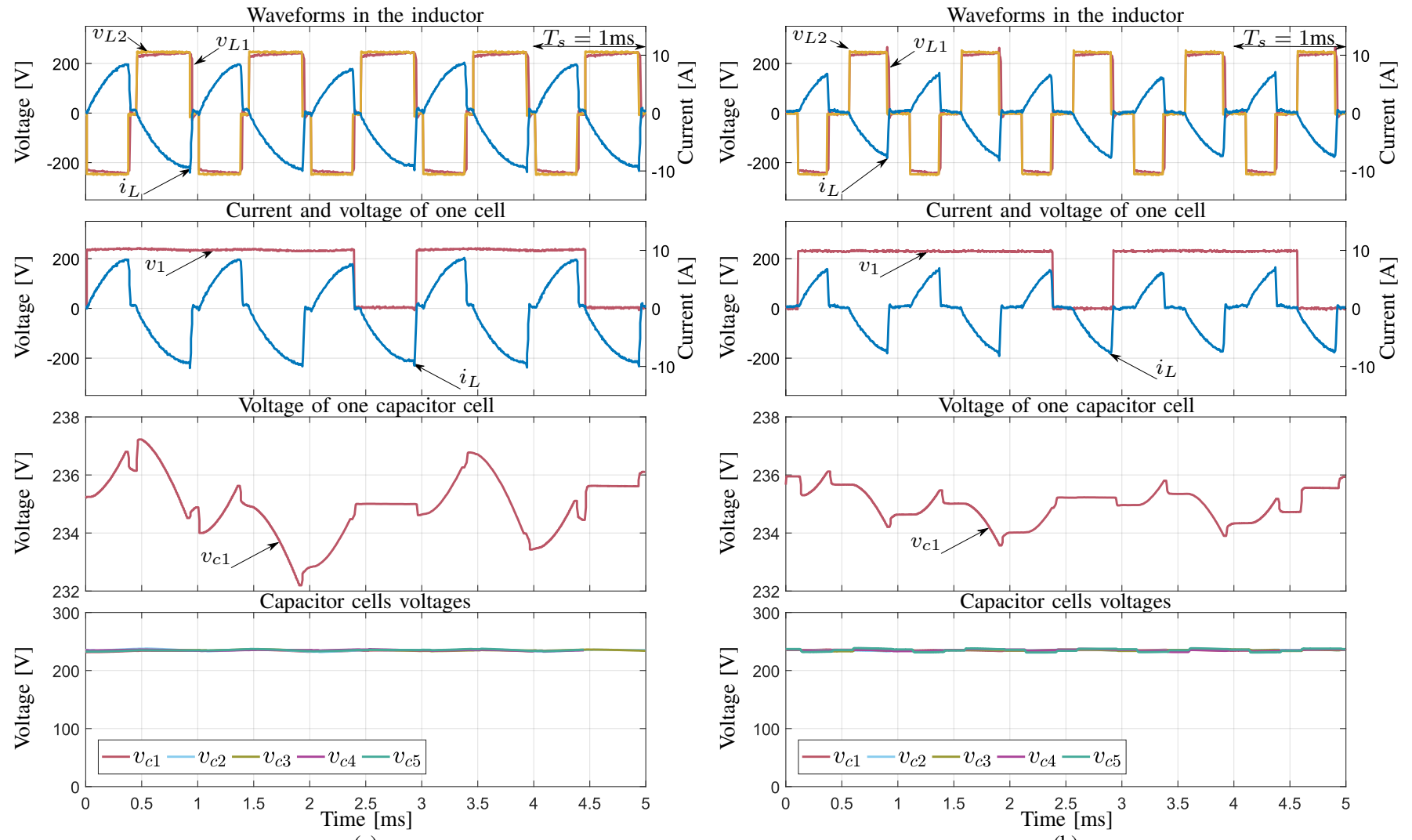

(a)

(b)

Fig. 11. Detailed experimental waveforms. (a) Waveforms at maximum power from low voltage to high voltage side. (b) Waveforms at $50 \%$ of maximum power from low voltage to high voltage side. 
from $210 \mathrm{~W}$ low to high side power transfer to $125 \mathrm{~W}$ high to low side power (Fig. 10 (b)), demonstrates the fast response and the bidirectional capacity of the converter. However, the reference step should occur at the end of the switching time to avoid undesired transients in the inductor current, generating a delayed response by $T_{s}$ at worst case.

The charge and discharge process of the cell capacitors can be observed in Fig. 10 (c), validating the voltage balance in the capacitors using the proposed operation. The ripple voltage in the capacitors is $1.6 \%$ at this operation point validating the design equation (13).

Voltage and current of one cell in the modulation time window are shown in Fig. 10 (d), verifying the soft-switching in the stack using the ATCM. The ratio of $\frac{N-2}{N}$ used in power balance condition is confirmed in this figure in the same way that was explained in the simulation subsection.

Detailed experimental waveforms at full power $\left(D_{1}=\frac{1}{2}\right)$ are illustrated in Fig. 11 (a), showing the voltages and current in the inductor, the current and voltage of one cell and all capacitor cell voltages. The same waveforms at $50 \%$ of maximum power $\left(D_{1}=\sqrt{\frac{1}{8}}\right)$ are shown in Fig. 11 (b), allowing the comparison between these two points of operation. In both cases, the experimental results validate the previous analysis for waveforms in the inductor, ZCS in half-bridge cells, capacitor voltage ripple and power balance.

The maximum efficiency of the prototype was $97.72 \%$ at $77 \%$ of maximum power transfer, and it remains over $97 \%$ in the $40-100 \%$ power transfer range as is shown in Fig. 9. At lower power transfer levels, the experimental efficiency decreases to a minimum of $92 \%$ because the losses independent of the power, such as those of the embedded measuring sensors, are increasingly relevant at lower power transfer levels. The experimental peak current is 1.98 times the average current at full power, and it increases as the power transfer decreases (gray line in Fig. 9) drawing a curve similar to the simulated in Fig. 5.

\section{CONCLUSIONS}

An asymmetric triangular current mode (ATCM) for a dc-dc MMC has been successfully validated, achieving bidirectional power control, ZCS and balance of the voltage in capacitor cells. A simple control law is proposed to control the converter. Theoretical analysis has been verified with full-scale simulations and a scaled-down prototype, showing higher efficiency $(97.72 \%)$ and lower peak current compared to previous reports of this topology using resonant operation. The experimental results confirm the operation principle, the voltage balance in the cells and the operation at a high number of cells and voltages. As a disadvantage, the asymmetric operation in a dcdc MMC with a low number of cells could saturate the core of the transformer if it is used for galvanic isolation. However, the asymmetry is reduced for high step ratio due to the need for a larger number of cells. With these results, the converter using ATCM has the potential to be a good solution for high step ratio dc-dc conversion.

\section{REFERENCES}

[1] P. Bresesti, W. L. Kling, R. L. Hendriks, and R. Vailati, "Hvdc connection of offshore wind farms to the transmission system," IEEE Transactions on energy conversion, vol. 22, no. 1, pp. 37-43, 2007.

[2] A. Lesnicar and R. Marquardt, "An innovative modular multilevel converter topology suitable for a wide power range," in 2003 IEEE Bologna Power Tech Conference Proceedings,, vol. 3, pp. 6-pp, IEEE, 2003.

[3] D. Pudney, "Hierarchical control in a 5-terminal vsc-hvdc project," in Energize Mag., 2014.

[4] S. Debnath, J. Qin, B. Bahrani, M. Saeedifard, and P. Barbosa, "Operation, control, and applications of the modular multilevel converter: A review," IEEE Transactions on Power Electronics, vol. 30, no. 1, pp. 37-53, 2015.

[5] S. P. Engel, M. Stieneker, N. Soltau, S. Rabiee, H. Stagge, and R. W. De Doncker, "Comparison of the modular multilevel dc converter and the dual-active bridge converter for power conversion in hvdc and mvdc grids," IEEE Transactions on Power Electronics, vol. 30, no. 1, pp. 124137, 2014

[6] J. D. Páez, D. Frey, J. Maneiro, S. Bacha, and P. Dworakowski, "Overview of dc-dc converters dedicated to hvdc grids," IEEE Transactions on Power Delivery, vol. 34, no. 1, pp. 119-128, 2018.

[7] R. W. De Doncker, D. M. Divan, and M. H. Kheraluwala, "A three-phase soft-switched high power density dc/dc converter for high power applications," in Conference Record of the 1988 IEEE Industry Applications Society Annual Meeting, pp. 796-805, IEEE, 1988.

[8] W. Chen, X. Ruan, H. Yan, and K. T. Chi, "Dc/dc conversion systems consisting of multiple converter modules: stability, control, and experimental verifications," IEEE Transactions on Power Electronics, vol. 24, no. 6, pp. 1463-1474, 2009.

[9] G. Ortiz, High-power DC-DC converter technologies for smart grid and traction applications. PhD thesis, ETH Zurich, 2014.

[10] S. Kenzelmann, A. Rufer, D. Dujic, F. Canales, and Y. R. De Novaes, "Isolated dc/dc structure based on modular multilevel converter," IEEE Transactions on Power Electronics, vol. 30, no. 1, pp. 89-98, 2014.

[11] A. Mohammadpour, L. Parsa, M. H. Todorovic, R. Lai, R. Datta, and L. Garces, "Series-input parallel-output modular-phase dc-dc converter with soft-switching and high-frequency isolation," IEEE Transactions on Power Electronics, vol. 31, no. 1, pp. 111-119, 2015.

[12] B. Zhao, Q. Song, J. Li, Q. Sun, and W. Liu, "Full-process operation, control, and experiments of modular high-frequency-link dc transformer based on dual active bridge for flexible mvdc distribution: a practical tutorial," IEEE Transactions on Power Electronics, vol. 32, no. 9, pp. 6751-6766, 2016.

[13] N. Denniston, A. M. Massoud, S. Ahmed, and P. N. Enjeti, "Multiplemodule high-gain high-voltage dc-dc transformers for offshore wind energy systems," IEEE Transactions on Industrial Electronics, vol. 58, no. 5, pp. 1877-1886, 2010.

[14] T. Lüth, M. M. Merlin, T. C. Green, F. Hassan, and C. D. Barker, "Highfrequency operation of a dc/ac/dc system for hvdc applications," IEEE Transactions on Power Electronics, vol. 29, no. 8, pp. 4107-4115, 2013.

[15] J. A. Ferreira, "The multilevel modular dc converter," IEEE Transactions on Power Electronics, vol. 28, no. 10, pp. 4460-4465, 2013.

[16] K. Filsoof and P. W. Lehn, "A bidirectional modular multilevel dcdc converter of triangular structure," IEEE Transactions on Power Electronics, vol. 30, no. 1, pp. 54-64, 2014.

[17] I. Gowaid, G. Adam, A. M. Massoud, S. Ahmed, D. Holliday, and B. Williams, "Quasi two-level operation of modular multilevel converter for use in a high-power dc transformer with dc fault isolation capability," IEEE Transactions on Power Electronics, vol. 30, no. 1, pp. 108-123, 2014.

[18] R. Vidal-Albalate, J. Barahona, D. Soto-Sanchez, E. Belenguer, R. S. Peña, R. Blasco-Gimenez, and H. Z. de la Parra, "A modular multilevel dc-dc converter for hvdc grids," in IECON 2016-42nd Annual Conference of the IEEE Industrial Electronics Society, pp. 3141-3146, IEEE, 2016.

[19] S. Cui, N. Soltau, and R. W. De Doncker, "A high step-up ratio softswitching dc-dc converter for interconnection of mvdc and hvdc grids," IEEE Transactions on Power Electronics, vol. 33, no. 4, pp. 2986-3001, 2017.

[20] X. Zhang, T. C. Green, and A. Junyent-Ferré, "A new resonant modular multilevel step-down dc-dc converter with inherent-balancing," IEEE Transactions on Power Electronics, vol. 30, no. 1, pp. 78-88, 2014.

[21] Y. Li, X. Lyu, and D. Cao, "A zero-current-switching high conversion ratio modular multilevel dc-dc converter," IEEE Journal of Emerging 
and Selected Topics in Power Electronics, vol. 5, no. 1, pp. 151-161, 2016.

[22] X. Xiang, X. Zhang, G. P. Chaffey, and T. C. Green, "An isolated resonant mode modular converter with flexible modulation and variety of configurations for mvdc application," IEEE Transactions on Power Delivery, vol. 33, no. 1, pp. 508-519, 2017.

[23] X. Zhang, M. Tian, X. Xiang, J. Pereda, T. C. Green, and X. Yang, "Large step ratio input-series-output-parallel chain-link dc-dc converter," IEEE Transactions on Power Electronics, 2018.

[24] D. Jovcic, "Bidirectional, high-power dc transformer," IEEE transactions on Power Delivery, vol. 24, no. 4, pp. 2276-2283, 2009.

[25] G. Ortiz, H. Uemura, D. Bortis, J. W. Kolar, and O. Apeldoorn, "Modeling of soft-switching losses of igbts in high-power high-efficiency dualactive-bridge dc/dc converters," IEEE Transactions on Electron Devices, vol. 60, no. 2, pp. 587-597, 2012.

[26] F. Jauch and J. Biela, "Generalized modeling and optimization of a bidirectional dual active bridge dc-dc converter including frequency variation," IEEJ Journal of Industry Applications, vol. 4, no. 5, pp. $593-$ 601, 2015.

[27] A. Hillers, D. Christen, and J. Biela, "Design of a Highly efficient bidirectional isolated LLC resonant converter," 15th International Power Electronics and Motion Control Conference and Exposition, EPE-PEMC 2012 ECCE Europe, vol. 1, no. 3, pp. DS2b.13-1-DS2b.13-8, 2012.

[28] X. Tan and X. Ruan, "Equivalence Relations of Resonant Tanks: A New Perspective for Selection and Design of Resonant Converters," IEEE Transactions on Industrial Electronics, vol. 63, no. 4, pp. 2111-2123, 2016.

[29] C. Pineda, J. Pereda, X. Zhang, and F. Rojas, "Triangular current mode for high step ratio modular multilevel dc-dc converter," in 2018 IEEE Energy Conversion Congress and Exposition (ECCE), pp. 5185-5189, IEEE, 2018.

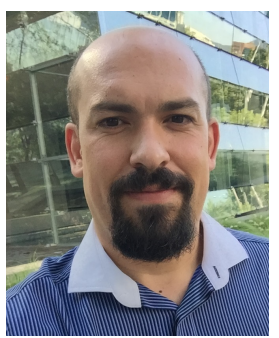

Cristian Pineda (S'18) received a B.Sc. in electrical engineering (Hons.) from the Universidad de Chile, Santiago, in 2010. Since 2017, he is a part-time lecturer at the Universidad de Santiago de Chile. He is currently pursuing the $\mathrm{PhD}$ degree with the Pontificia Universidad Católica de Chile and the University of Nottingham. His current research interests include power electronics and control applied to DC-DC power conversion, renewable energy and industrial applications.

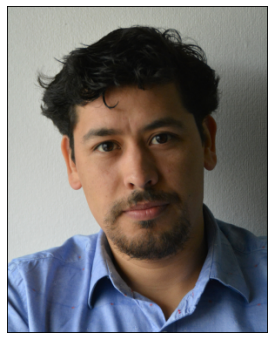

Felix Rojas (S'09-M'14) was born in Santiago, Chile. He received the B. Eng. and M.Sc. degrees in electrical engineering (Hons.) from the Universidad de Santiago de Chile, Santiago, in 2009, and the doctoral degree in electrical engineering from the Technical University of Munich, Munich, Germany, in 2015. He is currently an Assistant Professor of Electrical Engineering with the Universidad de Santiago de Chile, where is leading the Electrical Energy Technologies Research Center (E2TECH). $\mathrm{He}$ is also a Associate Research with the Solar Energy Research Center (SERC Chile). His research interests are in control of power electronics converters for solar and wind energy conversion, power networks conditioning, high power electric vehicles chargers, and variable speed drives.

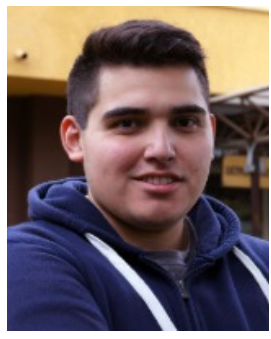

Carlos Cerda received a B.Eng. degree in electrical engineering from the Universidad de Santiago de Chile in 2018. He is currently a postgraduate student from the Universidad de Santiago de Chile and he joined to the Electrical Energy Technologies Research Center (E2TECH) at the same university. His research interests include DC-DC converters for electrical network drive and control for a softswitched mode in DC-DC converters.

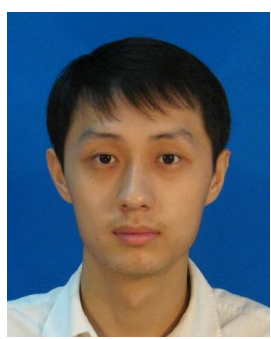

Xiaotian Zhang (S'11-M'12) was born in Xi'an, China, in 1983. He received the B.S. (with honors) and M.S. degrees in Electrical Engineering from Jiaotong University, Xi' an, China, in 2006 and 2009, respectively, and the $\mathrm{PhD}$ degree (with honors) in Electrical Engineering and Electronics from the University of Liverpool, U.K., in 2012.

Until 2015, he was with the Department of Electrical Engineering, Imperial College London, U.K. He is currently an Associate Professor of Department of Electrical Engineering, Xian Jiaotong University, China. His research interests include control and design of HVDC converters.

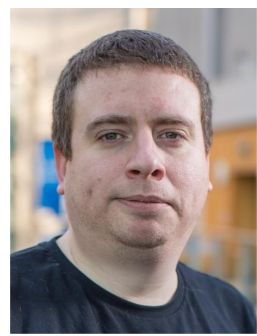

Alan Watson (S'03-M'08) received the M.Eng. degree (Hons.) in electronic engineering from the University of Nottingham, UK in 2004, and a PhD, also from the University of Nottingham in 2008. In 2009, he was a Research Fellow with the Power Electronics Machines and Control Group, University of Nottingham, where he was involved in the UNIFLEX project (http://www.eee.nott.ac.uk/uniflex/). Since 2009, he has been involved in various projects in the area of high power electronics including high power resonant converters, high voltage power supplies, and multilevel converters for grid connected applications such as HVDC and Flexible AC Transmission Systems. In 2012, he was promoted to Senior Research Fellow before becoming an Assistant Professor in High Power Electronics in 2013. His current research interests include the development and control of advanced high power conversion topologies for industrial applications. 